DOI: $10.15393 /$ j3.art.2021.9090

UDC 517.547

\author{
F. N. Garif'yanov, E. V. Strezhneva
}

\title{
ON THE DIFFERENCE EQUATION ASSOCIATED WITH THE DOUBLY PERIODIC GROUP AND ITS APPLICATIONS
}

\begin{abstract}
Let $D$ be a rectangle. We consider a four-element linear difference equation defined on $D$. The shifts of this equation are the generating transformations of the corresponding doubly periodic group and their inverse transformations. We search for a solution in the class of functions that are holomorphic outside $D$ and vanish at infinity. Their boundary values satisfy a Hölder condition on any compact that does not contain the vertices. At the vertices, we allow, at most, logarithmic singularities. The independent term is holomorphic on $D$, and its boundary value satisfies a Hölder condition. The independent term may not be analytically continuable across an interval of the boundary, since the solution and the independent term belong to different classes of analytical functions. We regularize the difference equation and determine the conditions for the regularization to be equivalent. If the independent term is an odd function, then the problem is solvable. Additionally, we give some applications of the difference operator to interpolation problems for integer functions of exponential type and the construction of biorthogonally conjugated systems of analytical functions.
\end{abstract}

Key words: difference equation, regularization method, biorthogonal systems, entire functions of exponential type

\section{Mathematical Subject Classification: 30D05}

1. Introduction. Let $D$ be a rectangle with vertices $t_{1}=-a-i$, $t_{2}=a-i, t_{3}=-t_{1}$, and $t_{4}=-t_{2}$, and sides $\ell_{j}, j=\overline{1,4}$, considered in the order in which they occur on the boundary $\Gamma=\partial D$. Here, we assume that $a \geqslant 1$ and $t \in \ell_{1} \Rightarrow \operatorname{Im}\{t\}=-1$. This is the fundamental region of the doubly periodic group with primitive periods $w_{1}=2 a$ and $w_{2}=2 i$ and generating transformations $\sigma_{k}(z)=z+w_{k}, k=1,2$.

(C) Petrozavodsk State University, 2021 
Consider the linear difference equation

$$
(V f)(z)=\sum_{j=1}^{4} f\left[\sigma_{j}(z)\right]=g(z), \quad z \in D
$$

where $\sigma_{3}=\sigma_{1}^{-1}, \sigma_{4}=\sigma_{2}^{-2}$, under the following assumptions:

1 ) The unknown function $f(z)$ is holomorphic outside $D$, and $f(\infty)=0$. Its boundary value $f^{-}(t)$ satisfies a Hölder condition on any compact that does not contain the vertices. At the vertices, we allow, at most, logarithmic singularities.

2) The independent term $g(z)$ is holomorphic inside $D$, and its boundary value $g^{+}(t) \in H_{\mu}(\Gamma)$.

We denote this class of solutions by $B$.

Note that, even though the operator $V$ commutes with the differentiation operator, we cannot apply to Equation (1) the powerful classical methods used in the study of convolution operators (see [9]). This is due to the fact that the set $C \backslash \bigcup \sigma_{j}(D), j=\overline{1,4}$, is disconnected. Equation (1) in the special case of a square $D$ was studied in [5]. The applications of the equation to interpolation problems for entire functions of exponential type (e.f.e.t.) were considered in [7]. The approximating properties of biorthogonal systems of analytical functions generated by Equation (1) were examined in the paper [6]. All the mentioned studies essentially relied on the symmetry $t \in \Gamma \Leftrightarrow i t \in \Gamma$. However, this is not the case if $a>1$, and the problems that arise in this case are of a different kind. For instance, instead of the classical lacunary Stieltjes moment problem for e.f.e.t., studied in the paper [7], we should consider the generalization of the problem to the case of two rays with a piecewise-exponential weight.

This paper consists of three sections. In the first section (2. Regularization of Equation (1)), we suggest a regularization method for Equation (1) and give the conditions that ensure the equivalence of the regularization. In the second section (3. On a special case), we prove that Equation (1) has only one solvability condition if $a \leqslant 3$. Some applications of these results are given in the third section (4. Some applications), where we consider applications to interpolation problems for e.f.e.t. in the case $a \leqslant 3$ and the approximating properties of biorthogonal systems of analytical functions generated by Equation (1) for $a<\sqrt{3}$. 
2. Regulariza quation (1). We search for a solution in the form of a Cauchy-type integral

$$
f(z)=\frac{1}{2 \pi i} \int_{\Gamma} \varphi(\tau)(\tau-z)^{-1} d \tau, \quad z \notin \bar{D}
$$

with an unknown density $\varphi(\tau)$. Let us define an odd involutive piecewiselinear shift $\alpha(t)=\left\{\sigma_{m}(t), t \in \ell_{m}\right\}$ that changes the orientation of $\Gamma$. We can assume, without loss of generality, that

$$
\varphi(t)+\varphi[\alpha(t)]=0 .
$$

Indeed, the density in the Cauchy-type integral (2) is defined up to a term $a^{+}(\tau)$, where $a(z)$ is a function holomorphic in $D$. Then, we consider condition (3) as an unconditionally solvable Carleman problem with respect to the unknown function $a(z)$ (see [3]). It follows from (2) that

$$
(1) \Leftrightarrow(E \varphi)(z) \equiv \frac{1}{2 \pi i} \int_{\Gamma} A(z, \tau) \varphi(\tau) d \tau=g(z), \quad z \in D,
$$

where

$$
A(z, \tau)=\sum_{j=1}^{4}\left(\tau-\sigma_{j}(z)\right)^{-1}
$$

If we proceed in (4) to the limit as $z \rightarrow t$, we obtain a formula similar to that of Sokhotski-Plemelj, namely

$$
\left(E^{+} \varphi\right)(t)=-2^{-1} \varphi(\alpha(t))+(E \varphi)(t)
$$

Therefore, according to condition (3), we have

$$
(T \varphi)(t) \equiv \varphi(t)+\frac{1}{2 \pi i} \int_{\Gamma} K(t, \tau) \varphi(\tau) d \tau=g^{+}(t)-g^{+}[\alpha(t)],
$$

where

$$
K(t, \tau)=A(t, \tau)-A[\alpha(t), \alpha(t)]
$$

and $(T \varphi)(t)=\left(E^{+} \varphi\right)(t)-\left(E^{+} \varphi\right)(\alpha(t))$.

Lemma 1. Kernel (7) is bounded. 
Proof. The proof boils down to a straightforward verification of the assertion of the lemma for all possible positions of the point $t$ on the sides of the rectangle.

So, we have regularized Equation (1). We now make the inverse transition from Fredholm equation (6) to Equation (1). Assume that Equation (6) is solvable. Then, there exists a solution with property (3). The proof is completely similar to the one given in the paper [1]. Thus, we have

$$
\left(E^{+} \varphi\right)(t)-\left(E^{+} \varphi\right)(\alpha(t))=g^{+}(t)-g^{+}[\alpha(t)] \Rightarrow(E \varphi)(z)=g(z)+C, \quad z \in D .
$$

Assume that Equation (6) has $N$ solvability conditions.

Theorem 1. Equation (1) has $N+1$ solvability conditions, namely $N$ solvability conditions of integral Equation (6) and the condition $(E \varphi)(0)=g(0)$, which ensures the equivalence of the regularization.

Let us formulate four statements that we will use later on.

1) Kernel (7) is skew-symmetric, i. e., $K(t, \tau)=-K(\tau, t)$.

2) The constant function is a solution of the associated equation

$$
T^{\prime} \psi=0
$$

3) A fundamental system of solutions (f.s.s.) of either Equation (8) or the homogeneous equation

$$
T \varphi=0
$$

can be constructed, such that some of the functions satisfy condition (3), while the other functions satisfy the opposite condition, i. e.,

$$
\varphi(t)=\varphi[\alpha(t)]
$$

The proof is totally similar to the one given in [1].

4) Kernel (5) fulfills the condition

$$
\frac{1}{2 \pi i} \int_{\Gamma} g^{+}(\tau) A(z, \tau) d \tau=0, z \in D,
$$

which means that it is not a generating kernel. Indeed, the transformations $\sigma_{j}(z), j=\overline{1,4}$, map the points $z$ of int $D$ into points of ext $D$.

Lemma 2. Assume that the f.s.s. of Equation (9) contains a function $\varphi(t)$ with property (10). Then, the function $\psi(t)=\varphi(t)+\left(E^{+} \varphi\right)(t)$ is a 
nontrivial solution of Equation (9) with property (10). Conversely, assume that the f.s.s. of Equation (8) contains a function $\psi(t)$ with property (3). Then the function $\varphi(t)=\psi(t)-\left(E^{+} \psi\right)(t)$ is a nontrivial solution of Equation (9) with property (10).

Proof. The equality $2 \varphi(t)=-\left(E^{+} \varphi\right)(t)-\left(E^{+} \varphi\right)[\alpha(t)]$ is true for the function $\varphi(t)$, which means that the function $\psi(t)$ has property (3). For such solutions, the associated equation can be written in the form $2 \psi(t)=\left(E^{+} \psi\right)(t)-\left(E^{+} \psi\right)[\alpha(t)]$, and the validity of the first part of the lemma can be proven by direct substitution. Assume that $\psi \equiv 0$. Then the solution of Equation (10) is the boundary value of a function that is analytical in $D$ and has property (10), i. e., it is the constant function, which is a solution of the associated equation. The second part of the lemma can be proven in a similar manner.

Corollary 1. The number of solutions with property (10) in the f.s.s. of Equation (9) is the same as the number of functions with property (3) in the f.s.s. of Equation (8). The number of solutions with property (3) in the f.s.s. of Equation (9) is the same as the number of functions with property (10) in the f.s.s. of the associated equation.

The statement in the second part of the corollary follows from that in the first part as a consequence of the Fredholm alternative, i. e., the f.s.s. of Equation (9) contains at least one function having property (3). In what follows, we assume that $\varphi(t)$ denotes a function with property (3), and $\psi(t)$ denotes a function with property (10).

Definition 1. If

$$
(E \varphi)(z)=C, \quad z \in D,,
$$

with $C \neq 0$, then we say that $\varphi(t)$ is a solution of the first kind.

Lemma 3. If $\varphi(t)$ is a solution of the first kind, then

$$
\int_{\Gamma} \varphi(\tau) d \tau=0
$$

Proof. Proceed to the limit in (11) as $z \rightarrow t$. As a result, obtain

$$
\frac{\varphi(t)}{2}+\frac{1}{2 \pi i} \int_{\Gamma} A(t, \tau) \varphi(\tau) d \tau=C .
$$


Multiply this equality by $\varphi(t)$ and integrate over $\Gamma$; then change the order of integration in the double integral. Taking into account that kernel (5) is skew-symmetric and, also, that $(10) \Leftrightarrow(12)$, obtain

$$
-C \int_{\Gamma} \varphi(t) d t=C \int_{\Gamma} \varphi(t) d t .
$$

This finishes the proof of the lemma.

Definition 2. If $\varphi(t)$ is a solution that satisfies condition (11) for $C=0$ and, at the same time, condition (12) does not hold, then we say that $\varphi(t)$ is a solution of the second kind.

Lemma 4. Equation (10) cannot have both a solution $\varphi(t)$ of the first kind and a solution $\varphi_{1}(t)$ of the second kind.

Proof. Assume the opposite. Multiply (13) by the function $\varphi_{1}(t)$ and integrate the obtained equality over $\Gamma$. Since the product of two functions with property (3) is a function with property (10), get

$$
0=C \int_{\Gamma} \psi_{1}(t) d t .
$$

This concludes the proof of the lemma.

Definition 3. If $\varphi(t)$ is a solution that satisfies condition (11) for $C=0$ and condition (12), then we say that $\varphi(t)$ is a solution of the third kind.

Let $\zeta(z)=\zeta(z ; 2 a ; 2 i)$ be a quasiperiodic Weierstrass zeta function with primitive periods $2 a$ and $2 i$ (see [8], part $2, \S 11$ ).

Lemma 5. Assume that Equation (9) has a solution $\varphi(t)$ of the third kind. Then the integral

$$
\psi(t)=\frac{1}{2 \pi i} \int_{\Gamma} \zeta(\tau-t) \varphi(\tau) d \tau,
$$

understood in the sense of the Cauchy principal value, is a nontrivial solution of the associated Equation (8) and it has property (10).

Proof. As a consequence of equality (12), the function given by (14) fulfills condition (10). For such solutions, we have

$$
(8) \Rightarrow\left(E^{+} \psi\right)(t)+\left(E^{+} \psi\right)(\alpha(t))=0 \Rightarrow(E \psi)(z)=0, z \in D .
$$


It remains to note that $\psi(t)=\psi^{+}(t)-\varphi(t)$ and verify straightforwardly that the lemma holds. If we assume that $\psi(t) \equiv 0$, then $\varphi(t)=\varphi^{+}(t)$, i. e., it follows, by property $(3)$, that $\varphi(t) \equiv 0$. This finishes the proof.

Conversely, assume that $\psi(t)$ is a function having property (10) and belonging to the f.s.s. of the associated equation. The Carleman problem $a^{+}(t)+a^{+}[\alpha(t)]=\psi(t)$ is solvable (see [3]). Then, the function $\varphi(t)=a^{+}(t)-a^{+}[\alpha(t)]$ is a solution of Equation (9) of the third kind. Here, we assume that the solution $\psi(t)$ is not a constant, i. e., it is not the boundary value of an analytical function.

Consider now the homogeneous Equation (9) and replace the variables $\tau$ and $t$ with $-\tau$ and $-t$, taking into account that the shift $\alpha(t)$ is an odd function. Therefore, $(T \varphi)(t)=0 \Leftrightarrow(T \varphi)(-t)=0$. The same is valid in the case of the associated Equation (8).

Corollary 2. The f.s.s. of Equation (9) or Equation (8) can be constructed in such a manner that some of the functions belonging to it are even and the rest are odd.

Corollary 3. Assume that $\varphi(t)$ is either an even function having property (3) or an odd function having property (10). Then,

$$
\forall j \int_{\ell_{j}} \varphi(\tau) d \tau=0 .
$$

Corollary 4. Assume that $g(z)$ is an odd function and the nonhomogeneous Equation (6) is solvable. Then, there exists an odd solution $\varphi(t)$ of Equation (6), and the condition of equivalence of the regularization in Theorem 1 is automatically fulfilled.

Let us consider another zeta function $\zeta_{1}(z)=\zeta(z ; 2 a-2 i ; 2 a+2 i)$. Note that it is not defined on the sides of the rectangle but on its diagonals. Note also that the differences $\tau-t$ and $\alpha(\tau)-\alpha(t)$ differ from each other by a quasiperiod of the function $\zeta_{1}(z)$. The value of this quasiperiod depends on the relative position of the points $\tau$ and $t$ on the sides of the rectangle. Thus, $\zeta(\tau-t)-\zeta[\alpha(\tau)-\alpha(t)]=\beta(t, \tau)$, where $\beta(t, \tau)$ is a piecewise-constant function discontinuous at the vertices with respect to each variable. Kernel (5) is the sum of the first four terms of the expansion of the function $\zeta_{1}(\tau-z-2 a)$ in a series of partial fractions.

Remark 1. If we consider the kernel $\zeta_{1}(\tau-z-2 a)$ instead of kernel (5), then, by the foregoing, the research of integral Equations (8) and (9) does 
not present difficulties (on this matter, see [4]). Kernel (5) is convenient because it has the minimum number of terms for which the given reasoning is valid.

Lemma 6. Let us suppose that $T \varphi=0$. If the solution $\varphi(t)$ is an even function with property (3), then $T \varphi^{\prime}=0$.

Proof. Consider the kernel $B(z, \tau)=\zeta_{1}(\tau-z-2 a)-A(z, \tau)$. Then, $\varphi(t)-G^{+}(t)+G^{+}[\alpha(t)]=0$, where

$$
G(z)=\frac{1}{2 \pi i} \int_{\Gamma} B(z, \tau) \varphi(\tau) d \tau, \quad z \in D
$$

Note that

$$
\int_{\Gamma} \beta(t, \tau) \varphi(\tau) d \tau=0
$$

by virtue of Corollary 3. Here, $G(z) \in A[D] \Rightarrow G^{+}(t) \in C^{1}(\Gamma)$. Moreover, $G^{+}[\alpha(t)] \in C(\Gamma)$, since the solution is an even function. It only remains to note that $\partial A / \partial z=-\partial A / \partial \tau$ and

$$
\frac{d}{d z}(E \varphi)(z)=-\frac{1}{2 \pi i} \int_{\Gamma} \varphi(\tau) \frac{\partial A(z, \tau)}{\partial \tau} d \tau=\left(E \varphi^{\prime}\right)(z), z \in D,
$$

if we integrate by parts. In this case, the term that appears outside the integral vanishes because it is the increment of a continuous function along a closed curve. This concludes the proof.

Corollary 5. The function $\varphi^{\prime}(t)$ from Lemma 6 is an odd solution of the third kind.

3. On a special case. Unfortunately, it is hardly possible to determine the number of solvability conditions of Equation (1) in the general case. However, this turns out to be possible in the particular case, in which $a \leqslant 3$, by using the contraction mapping theorem in a Banach space. For the sake of simplicity, we assume that $a=3$. Consider an odd solution of the associated equation with property (10). Suppose that

$$
M=\max |\psi(t)|, \quad t \in \Gamma \text {. }
$$

It is enough to consider just two cases. We will find an upper estimate for the absolute value of the integral term in Equation (8). 
I. Equality (15) is attained at some point $t \in \ell_{1}$. There are three possible subcases:

a) $\tau \in \ell_{1} \Rightarrow K(t, \tau)=0$.

b) $\tau \in \ell_{3}$. In this case, we have $K(t, \tau)=(u+2 i)^{-1}+(u-6)^{-1}+$ $(u+6)^{-1}-(u-6 i)^{-1}-(u-6-4 i)^{-1}-(u+6-4 i)^{-1}$, where $u=\tau-t$. Since $u=2 i+\gamma$, with $\gamma \in[-6,6]$, we obtain $K(t, \tau)=-i f(x)$, where $f(x)=8\left[(x+16)^{-1}+(x+40)\left(x^{2}-64 x+1600\right)^{-1}\right]$ and $x=\gamma^{2} \in[0,36]$. By Corollary 3, we take the number $2^{-1}[\max f(x)-\min f(x)] \leqslant 0.27$ instead of $\max f(x)$.

c) $\tau \in L$, where $L=\ell_{2} \cup \ell_{4}$. According to condition (10), we have

$$
\int_{L} K(t, \tau) \psi(\tau) d \tau=\int_{\ell_{4}} K_{1}(t, \tau) \psi(\tau) d \tau,
$$

where $K_{1}(t, \tau)=(u+2 i)^{-1}+(u-6)^{-1}-(u+6-4 i)^{-1}-(u+12-$ $-2 i)^{-1}-(u+6+2 i)^{-1}-(u+12)^{-1}+(u-4 i)^{-1}+(u-6-2 i)^{-1}$. Since $t=-i+\beta,-3 \leqslant \beta \leqslant 3$, and $\tau=-3+\gamma i,-1 \leqslant \gamma \leqslant 1$, we obtain $\left|K_{1}\right| \leqslant 1.14$. Furthermore, $6 \cdot 0,27+2 \cdot 1,14<2 \pi$, which means that $\psi \equiv 0$. Thus, the assumption was incorrect. There remains the last possibility.

II. Equality (15) is attained at some point $t \in \ell_{2}$. There are three possible subcases:

a) $\tau \in \ell_{2} \Rightarrow K(t, \tau)=0$.

b) $\tau \in \ell_{4}$. In this case, we have $K(t, \tau)=(u-2 i)^{-1}+(u+2 i)^{-1}+$ $+(u-6)^{-1}-(u+12-2 i)^{-1}-(u+12+2 i)^{-1}-(u+18)^{-1}$. Since $u=-6+\gamma i$, with $|\gamma| \leqslant 2$, we obtain $K(t, \tau)=-f(x)$, where $f(x)=24\left[(x+144)^{-1}+(x+40)\left(x^{2}+76 x+1600\right)^{-1}\right]$ and $x=\gamma^{2} \in[0,4]$. By Corollary 3 , we take the number $2^{-1}[\max f(x)-\min f(x)] \leqslant 0.04$ instead of $\max f(x)$.

c) $\tau \in L$, where $L=\ell_{1} \bigcup \ell_{3}$. According to condition (10), we have

$$
\int_{L} K(t, \tau) \psi(\tau) d \tau=\int_{\ell_{1}} K_{1}(t, \tau) \psi(\tau) d \tau,
$$

where

$K_{1}(t, \tau)=(u-2 i)^{-1}+(u-6)^{-1}-(u+4 i+6)^{-1}-(u+12+2 i)^{-1}-$ $-(u+4 i)^{-1}-(u+2 i-6)^{-1}+(u+6-2 i)^{-1}+(u+12)^{-1}$. Since $\tau=\gamma-i$, $|\gamma|<3$, and $t=3+x i,|x|<1$, we obtain $\left|K_{1}\right| \leqslant 0,95$. Furthermore, 
$2 \cdot 0,04+6 \cdot 0,95<2 \pi$, which means that $\psi \equiv 0$. Next, assume that the f.s.s. of the associated equation contains a nonconstant even function with property (10). Therefore, the f.s.s. of Equation (9) contains an even function $\varphi(t)$ with property (3). It follows from Corollary 5 that there exists an odd solution of the third kind, i. e., the f.s.s. of the associated equation contains an odd function with property (10) (see Lemma 5). Thus, we come to a contradiction with the estimates obtained above.

Remark 2. The foregoing estimates for the absolute values of the integrals worsen very quickly as a increases. The optimum estimate is the one obtained for $a=1$, i. e., when $D$ is a square. From a geometric point of view, this is obvious: the "more" the rectangle shape deviates from that of a square, the worse are the estimates.

Let us state the result we have obtained.

Lemma 7. If $a \in[1,3]$, then the f.s.s. of the associated equation contains only one function with property (10), namely the constant function.

Remark 3. The estimates that ensure the truth of Lemma 2 imply that the number a may be slightly greater than 3. In this paper, however, we do not dwell on the issue of how much greater.

Remark 4. It follows from the given estimates that the f.s.s. of the associated equation does not contain any function with property (3), and the f.s.s. of Equation (9) does not contain any function with property (10). We do not dwell on this issue in more detail since the solutions of the associated equation having property (3) are of little interest. They are automatically orthogonal to the right-hand side of Equation (6). The number of solvability conditions of Equation (1) does not depend on whether such solutions exist.

Theorem 2. If $a \in[1,3]$, then $N=0$ in Theorem 1, i.e., Equation (1) has only one solvability condition. Under the additional assumption that the independent term $g(z)$ is an odd function, Equation (1) is unconditionally solvable.

Remark 5. The ultimate form of the f.s.s. of Equations (8) and (9) can be made more specific if $a \in[1,3]$. In this case, the f.s.s. of Equation (8) contains only the constant function, and the f.s.s. of Equation (9) contains only an odd solution $\varphi_{0}(t)$ of the second kind, which has nonremovable discontinuities of the first kind (jump discontinuities) at least at two opposite vertices. 
Indeed, if one assumes that $\varphi_{0}(t) \in C(\Gamma)$, then $T \varphi_{0}=0 \Rightarrow T \varphi_{0}^{\prime}=0$, thus arriving at a contradiction.

4. Some applications. Consider an even e.f.e.t. $F(z)$ that is Borelassociated with an odd solution $f(z) \in B$ (see [2], §1, 1.1.). Its conjugated indicator diagram is, generally speaking, a rectangle $D$. Assume that

$$
g(z)=-2 \sum_{k=0}^{\infty} \frac{\gamma_{k} z^{2 k+1}}{(2 k+1) !}
$$

and the convergence radius of this series satisfies the condition $R>\sqrt{a^{2}+1}$. Since the solution is odd, we have

$$
(V f)(z)=-2 \int_{0}^{\infty} F(x) \exp (-2 a x) \operatorname{sh}(x z) d x-2 \int_{L} F(t) \exp (2 i t) \operatorname{sh}(t z) d t
$$

where $L$ is the ray $\arg t=\pi / 2$. Equate the Maclaurin coefficients in the expansions of the functions $g(z)$ and $(V f)(z)$. As the result, obtain

$$
\int_{0}^{\infty} F(x) x^{2 k+1} \exp (-2 a x) d x+\int_{L} F(t) t^{2 k+1} \exp (2 i t) d t=\gamma_{k}, k=\overline{0, \infty} .
$$

Theorem 3. If $a \in[1,3]$, then the lacunary moment problem (16) is solvable. The corresponding homogeneous moment problem (for all $k$ and $\gamma_{k}=0$ ) has only one nontrivial solution $F_{0}(z)$.

Note that $F_{0}(z)$ is an upper function that is Borel-associated with the lower function $f_{0}(z)$, i.e., the Cauchy-type integral (2) with the density $\varphi_{0}(\tau)$ (see Remark 5).

Remark 6. The conjugated indicator diagram may not be the rectangle $D$ but some smaller convex set $D^{\prime} \subset D$. This case, however possible, is of little interest. Indeed, the independent term must allow the analytical continuation from $D$ to some neighborhood of infinity, and, moreover, $g(0)=0$. Provided that all these conditions hold, problem (1) is overdetermined. Then, $F(z)=G(z) / P(z)$, where $G(z)$ is an upper function that is Borel-associated with the lower function $g(z)$, and

$$
P(z)=\sum_{k=1}^{4} \exp \left[\sigma_{k}(0) z\right]
$$


is the characteristic quasipolynomial of the difference equation. The fraction $F(z)$ must be an e.f.e.t., and its lower function $f(z)$ must be a solution of Equation (1) from class B. It is obvious that such overdetermined problems are of little interest.

In conclusion, let us consider some properties of the biorthogonal conjugated systems of analytical functions generated by the difference operator $V$. In what follows, we assume that $a<\sqrt{3}$. Let us suppose that $A(\tau)=A(0, \tau)$

$$
\left\{\varphi_{m}(\tau)\right\}:\left(E \varphi_{m}\right)(z)=(-1)^{m+1}(m !)^{-1}\left(z^{m}-h^{m}\right), z \in D, m=0,1, \ldots,
$$

and $f_{m}(z)$ is the Cauchy-type integral (2) with the density $\varphi_{m}(\tau)$. Assume that $h_{0}=1$, and choose the constants $h_{m}$ for any $m>0$ in such a manner that the equivalence condition of the regularization in Theorem 1 holds. Carry out the normalization:

$$
\int_{\Gamma} f_{0}(\tau) d \tau=2 \pi i, \quad \forall m>0 \int_{\Gamma} f_{m}(\tau) d \tau=0 .
$$

Then the system of functions $\left\{f_{m}(z)\right\}, m=0,1, \ldots$, and

$$
A_{k}(z)=\left\{1, k=0 ; A^{(k)}(z), k \geqslant 1\right\}
$$

are biorthogonal on $\Gamma$ in the sense that

$$
\frac{1}{2 \pi i} \int_{\Gamma} f_{m}^{-}(\tau) A_{k}(\tau) d \tau=\delta_{m, k} .
$$

Consider the region $D_{0}$ limited by arcs of the four circles $\left|z-\sigma_{j}(0)\right|=$ $=\sqrt{a^{2}+1}, j=\overline{1,4}\left(0 \in D_{0}\right)$. The functions $f_{m}(z)$ are analytically continuable from the sides of $\Gamma$ to $\Gamma_{0}=\partial D_{0}$. Therefore, we can replace the curve $\Gamma$ in (18) with $\Gamma_{0}$. The region $D_{0}$ and its complement $\complement D_{0}$ are the only natural regions of convergence of the corresponding biorthogonal series. A number of theorems on their properties can be obtained in the same way as it was done in [6], where the case $a=1$ was considered. By way of illustration, we state here one of these theorems:

Theorem 4. Let $h(z)$ be an even function, such that $h(z) \in A\left[\complement D_{0}\right]$. Then,

$$
h(z)=\sum_{m=0}^{\infty} \alpha_{m} f_{m}(z), \quad z \in \complement D_{0},
$$


where the series converges absolutely and uniformly. The series coefficients are determined by biorthogonality condition (18).

Remark 7. Assume that $a=\sqrt{3}$. The set $D_{0}$ of points outside the four circles splits into two regions sharing a single boundary point, namely $z=0$. If $a>\sqrt{3}$, then the regions have no common boundary points.

\section{References}

[1] Aksent'eva E. P., Garif'yanov F. N. On the analysis of an integral equation with Carleman kernel. Soviet Mathematics, 1983, vol. 27, no. 4, pp. 53-63.

[2] Bieberbach L. Analytische Fortsetzung. Springer-Verlag, Berlin-Göttingen-Heidelberg, 1955.

[3] Chibrikova L. I. Boundary-value problems for rectangle. Uchen. Zap. Kazan Univ., 1963, vol. 123, no. 10, pp. 3-4.

[4] Garif'yanov F. N. Absolutely representing systems of elliptic functions. Mat. Zametki, 1996, vol. 59, no. 6, pp. 932-936.

[5] Garif'yanov F. N. Inversion problem for singular integral and difference equations for functions analytical outside a square. Russian Mathematics, 1993, vol. 37, no. 7, pp. 5-14.

[6] Garif'yanov F. N. Transformations of biorthogonal systems and some of their applications. II. Izv. Vyssh. Uchebn. Zaved. Mat., 1996, no. 8, pp. $13-24$.

[7] Garif'yanov F. N. Stieltjes Moments of Entire Functions of Exponential Type. Math. Notes, 2000, vol. 67, no. 5, pp. 572-576.

DOI: https://doi.org/10.1007/BF02676327

[8] Hurwitz A., Courant R. Vorlesungen über allgemeine Funktionentheorie und elliptische Funktionen. Springer-Verlag, Berlin-Göttingen-HeidelbergNew York, 1964. DOI: https://doi.org/10.1007/978-3-662-00750-1

[9] Napalkov V. V. Convolution Equations in Multidimensional Spaces. Nauka, Moscow, 1982.

Received September 11, 2020.

In revised form, September 18, 2020.

Accepted November 25, 2020.

Published online December 8, 2020. 
Garif'yanov F. N.

Kazan State Power Engineering University

51 Krasnosel'skaya st., Kazan 420066, Russia

E-mail: f.garifyanov@mail.ru

Strezhneva E. V.

Kazan National Research Technical University named after A. N. Tupolev

10 K. Marx st., Kazan, 42011, Russia

E-mail: strezh@yandex.ru 